\title{
UTILIZACIÓN DE LA QUÍMICA COMPUTACIONAL: MÉTODO SEMIEMPÍRICO PM3, PARA ELUCIDAR LA ESTRUCTURA DEL COMPLEJO BIS (1,5- DIFENIL-1,2, 4-TRIAZOL-3-TIONATO) PLOMO (II) $\left(\mathrm{Pb}(\mathrm{DTT})_{2}\right)$.
}

\author{
Jorge R. Angulo-Cornejo ${ }^{a^{*}}$; Carlo F. Tovar \\ RESUMEN
}

El estudio teórico por el método semiempírico PM3 ha sido utilizado para predecir la estructura más probable del Bis (1,5- difenil-1,2, 4-triazol-3-tionato) plomo (II) $\left(\mathrm{Pb}(\mathrm{DTT})_{2}\right)$, a través de la aplicación del Principio Máxima dureza de Pearson.

Se propuso tres posibles estructuras las cuales son reportadas por Angulo y colaboradores ${ }^{1}$, sobre la base de datos experimentales. Concluyendo luego del análisis de los cálculos teóricos que la estructura más probable es la $\Psi$ bipiramide trigonal en el que el plomo se coordina con dos ligando a través del azufre y el nitrógeno hidrazínico, coincidiendo con lo encontrado experimentalmente.

Palabras clave: Método semiempírico PM3, principio de máxima dureza, plomo (II), complejo, triazol, tionato.

\section{USING COMPUTATIONAL CHEMISTRY: SEMIEMPIRICAL Pm3 METHOD, TO ELUCIDATE THE STRUCTURE OF BIS (1,5-DIPHENYL-1,2,4-TRIAZOLE-3-THIONATE) LEAD(II) $\left(P b(D T T)_{2}\right)$.}

\begin{abstract}
This theoretical study at the level PM3, has been to predict the more stable structure of bis (1,5-diphenyl-1,2,4-triazole-3-thionate) lead (II) $\left(\mathrm{Pb}(\mathrm{DTT})_{2}\right)$, through the application of the Pearson's Principle Maximum Hardness (PMH).

Proposed three possible structures which are reported by Angulo et $\mathrm{al}^{1}$, to the based on experimental data. Determined that the structure presented greater hardness and thermodynamics stability is when lead atom is coordinated with the sulfur atom and the hydrazine nitrogen, in $\Psi$ bipiramide trigonal structure, coinciding this with the experimental data.
\end{abstract}

Key words: Semiempirical method PM3, principle maximum hardness, lead (II), complex, triazole, thionate.

\section{INTRODUCCIÓN}

Una de las tareas de la química computacional es la determinación de estructuras y propiedades moleculares mediante el uso de mecánica molecular, métodos semiempíricos y teorías de orbitales moleculares "a primeros principios" (ab initio) ${ }^{2}$

\footnotetext{
${ }^{a^{*}}$ Facultad de Química e Ingeniería Química, Universidad Nacional Mayor de San Marcos, Ciudad Universitaria,Av. Venezuela s/n Pab. B, Lima 100, Perú. joracperu@yahoo.es
} 
La gran dificultad para realizar cálculos más exactos como el método ab initio y DFT (Teoría del funcional de densidad) para el estudio de moléculas de mayor tamaño han sido los métodos aproximados llamados semiempíricos, esto debido a las restricciones tecnológicas existentes ${ }^{3}$. Entre los métodos semiempíricos desarrollados se encuentra el método PM3 (Parameterized Model number 3$)^{4}$. Este método surgió por la reparametrización del método AM1 (Modelo Austin 1, llamado así por la Universidad de Texas en Austin), y está parametrado para los elementos: H, C, Si, Ge, Sn, Pb, N, P, As, Sb, Bi, O, S, Se, Te, F, Cl, Br, I, Al, Ga, In, Tl, Be, Mg, $\mathrm{Zn}, \mathrm{Cd}, \mathrm{Hg}^{5}$.

El método PM3 toma la energía de valencia molecular $v_{\text {val }}$, que incluye la repulsión internuclear, como la suma de la energía puramente electrónica $\mathrm{E}_{e l, v a l}$, más la energía de la repulsión core-core $V_{c c}$ :

$$
U_{v a l}=E_{a l, w a i}+V_{c o}
$$

Los métodos tipo Dewar (MINDO, AM1, PM3) tratan a la molécula como una colección de electrones de valencia y centros atómicos, donde cada centro (core) consiste de un átomo y sus electrones internos. La aproximación más simple se puede escribir de la siguiente forma:

$$
V_{c E}=\sum_{B>A} \sum_{A}\left[C_{A} C_{B}\left(s_{A} s_{A} \mid s_{E} s_{B}\right)+f_{A E}\right]
$$

Donde $C_{A} C_{B}$ son las cargas del core A y B, además $\left(S_{A} S_{A} \mid S_{B} S_{B}\right)$ es la integral de repulsión electrónica que involucra los orbitales de valencia $s$ de los átomos A y B; el último término $f_{A B}$ corresponde a un valor que varía según el método y que concuerda con los datos experimentales.

El cálculo de la entalpía para los métodos tipo Dewar, entre ellos el PM3, se obtienen mediante:

$$
\Delta H_{f, 298, M(g)}^{0}=N_{A} U_{\theta l, w a i, M}-N_{A} \sum_{i} E_{V a l, A}+\sum_{i} \Delta H_{f, 298, A(\theta)}^{0}
$$

Donde $\Delta \mathrm{H}_{\mathrm{f}, 298, \mathrm{M}(\mathrm{g})}^{0}$ y $U_{e l, \mathrm{val}, \mathrm{M}}$ son valores experimentales que se toma de tablas termodinámicas ${ }^{6}$. Para mayor información sobre el formalismo del método semiempírico PM3 consultar las siguientes bibliografías ${ }^{7,8}$.

R. G Parr y W. Yang ${ }^{9}$ introdujeron conceptos químicos cualitativos que correlacionan la electronegatividad y dureza por medio de la teoría del funcional de densidad. El concepto de electronegatividad fue introducido primero por Pauling ${ }^{10}$ y la idea de dureza la propuso Pearson ${ }^{11}$ en el contexto de la teoría de ácidos y bases duras(os) y blandas(os) (HSAB).

La dureza puede ser considerada como una medida de la resistencia a la transferencia de carga. Variar el número de electrones en un sistema duro tiene un costo energético y por tanto este sistema tendrá poca tendencia a dar o aceptar electrones. 
La definición operacional de la dureza ha sido obtenida ${ }^{12}$ por una aproximación diferencial finita propuesta en la siguiente ecuación:

$$
\eta=\frac{I-A}{2} \cong \frac{z_{\operatorname{LNO}}-s_{\operatorname{HOM} D}}{2}
$$

Asumiendo la validez del Teorema de Koopmma's ${ }^{13}$ :

$$
-g_{\text {HOMO }}=I \quad-g_{L U M O}=A
$$

Donde ${ }^{\varepsilon} H O M O$ es la energía del orbital molecular ocupado más alto y ${ }^{\varepsilon} L U M O$ es la energía del orbital molecular desocupado más bajo.

La presencia de una buena separación del HOMO y LUMO en las moléculas ha sido asociada con una estabilidad estructural y cinética ${ }^{14}$, mientras que cuando la diferencia de energía HOMO-LUMO es pequeña es de esperar que el sistema sea poco estable y altamente reactivo ${ }^{15}$. Pearson postuló que las moléculas que tienen una separación entre HOMO y LUMO grande tienden a ser por regla natural lo más duras posibles e incrementan su estabilidad ${ }^{16}$.

\section{Detalles computacionales}

Se ha determinado la dureza $(\eta)$, la energía HOMO, energía LUMO, energía total del sistema a $0 \mathrm{~K}$ y a $298 \mathrm{~K}$ y el calor de formación, de las geometrías optimizadas de cada una de las tres posibles estructuras de la figura 1. Los estudios computacionales fueron realizados por medio de cálculos semiempíricos a nivel PM3 que se incluyen en el paquete de programas Hyperchem 8.0 Profesional $^{17}$, implementado en una computadora personal Intel (R) Atom N450 1.66Ghz, $1 \mathrm{~Gb}$ de RAM.

a)

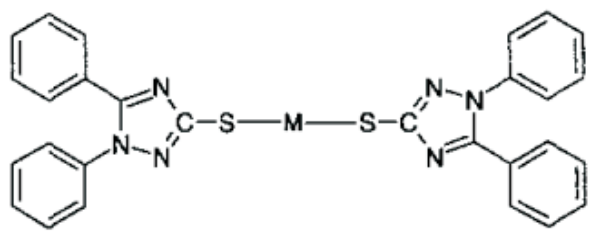

b)

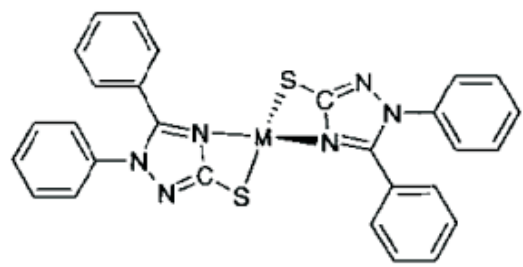

c)

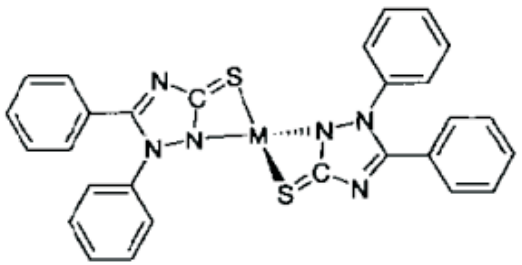

Figura 1. Las tres posibilidades de coordinación del ligando al metal 


\section{RESULTADOS Y DISCUSIÓN}

Los datos experimentales ${ }^{1}$ indican que el complejo de plomo (II) tiene una estereoquímica 1:2 (metal: ligando).

El espectro IR del ligando muestra las bandas de absorción a 1250 y $2507 \mathrm{~cm}^{-l}$ que corresponde a la tensión $v(C=S)$ y $v(\mathrm{~S}-\mathrm{H})$; asimismo, se puede notar un hombro ancho que corresponde a la señal del grupo $\mathrm{NH}$ a $3250 \mathrm{~cm}^{-1}$, lo que demuestra la coexistencia de dos formas tautoméricas con intercambio rápido del protón. En el espectro IR del complejo de plomo, se puede distinguir la desaparición de la señal del grupo NH y el grupo SH. En cambio la señal del $\mathrm{C}=\mathrm{S}$ se observa desplazado ligeramente a $1237 \mathrm{~cm}^{-1}$, confirmando de esta manera la coordinación del plomo con el ligando a través de los átomos de $\mathrm{S}$ y del nitrógeno hidrazínico que se desprotona. En la figura 2, en el rango entre 3071 y $2729 \mathrm{~cm}^{-1}$ del espectro del ligando se observa una serie de bandas de absorción de estiramiento que corresponde a $\mathrm{C}_{\mathrm{Ph}}-\mathrm{H}^{18,19,20,21}$ debido a la resonancia de los enlaces conjugados dentro del anillo aromático y el anillo heterocíclico. En el espectro del complejo de plomo (II), sólo se observa una señal definida a $3068 \mathrm{~cm}^{-1}$ debido a que la formación del nuevo enlace entre el plomo y los átomos dadores $\mathrm{S}$ y $\mathrm{N}$ que genera un anillo quelato de cuatro miembros provoca la desaparición de la resonancia entre los enlaces conjugados y el anillo triazol.
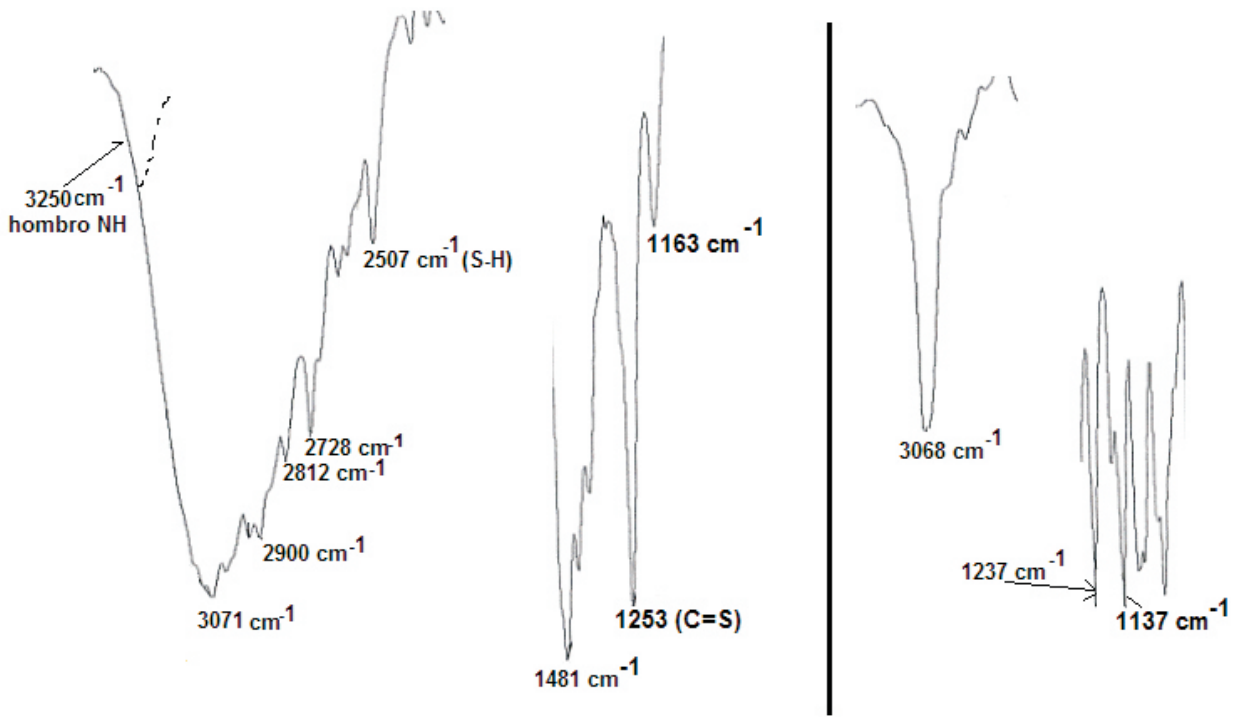

Figura 2. Espectro IR del ligando (izquierda) y del complejo de plomo (derecha)

En los espectros de $R M N-H^{l}$ a diferentes temperaturas $\left(-70^{\circ} \mathrm{C}-60^{\circ} \mathrm{C}\right)$ en $C D C l_{3}$ del ligando, se observa la presencia de dos formas tautoméricas. Se observa por ejemplo a $60^{\circ} \mathrm{C}$, la señal del protón $\mathrm{NH}$ a 12.45 ppm y mientras que el protón $\mathrm{CSH}$ es detectado a 1.55 ppm.

En el complejo de plomo no se observa la señal correspondiente al grupo S-H, ni la señal del protón N-H. Tomando en cuenta esto se puede deducir que el ligando debe haberse coordinado al metal a través del grupo C-S y el átomo de nitrógeno hidrazínico con pérdida del protón. (figura 3) 


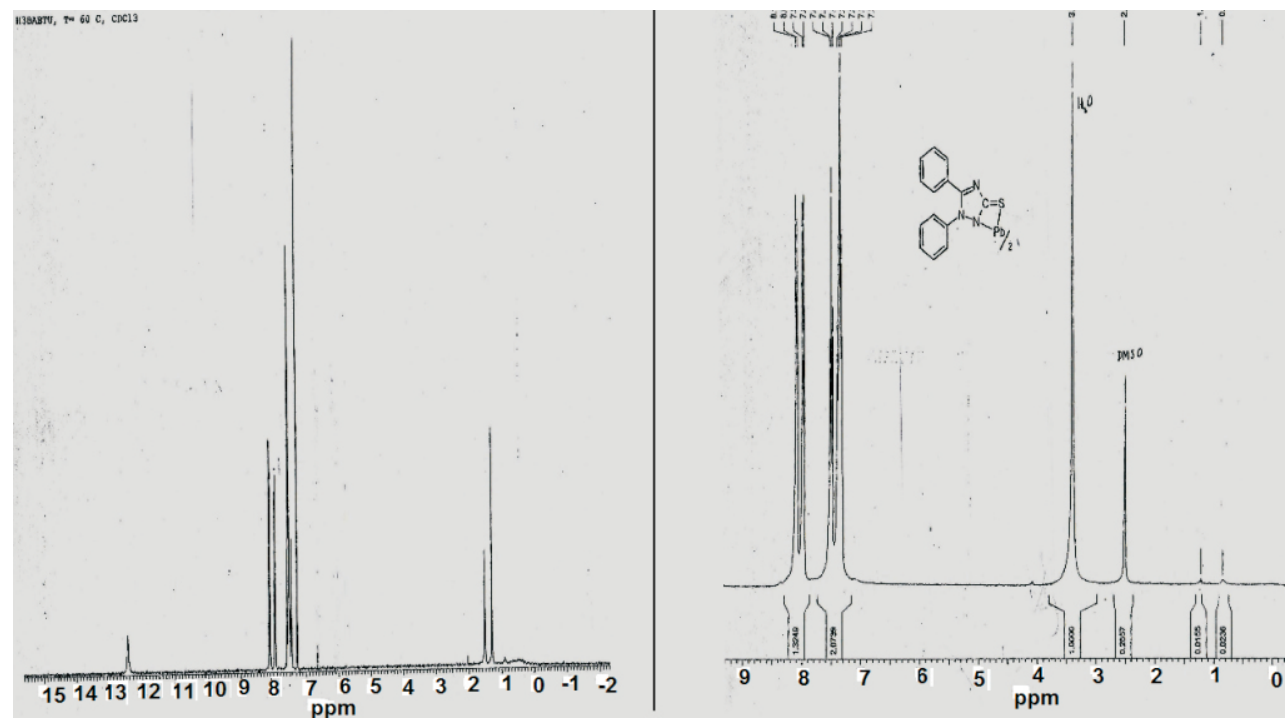

Figura 3. Espectros $\mathrm{RMN}-\mathrm{H}^{1}$ del ligando a $60^{\circ} \mathrm{C}$ (izquierda) y del complejo de plomo (derecha)

En el espectro $C^{13}\left(H^{1}\right)$ se nota un desplazamiento a campo bajo del grupo $\mathrm{C}=\mathrm{N}$ (de 149,7 a $158,6 \mathrm{ppm}$ ) típico de estructuras en donde el grupo $\mathrm{C}=\mathrm{N}$ no está en conjugación con otros dobles enlaces. La transición del grupo $\mathrm{C}=\mathrm{S}$ se desplaza a campo alto (de 166,4 a 161,7 ppm), ganando cierta protección debido a que la densidad electrónica, en ausencia de la capacidad de resonancia, se concentra en los átomos de $\mathrm{N}-\mathrm{C}-\mathrm{S}$ y $\mathrm{Pb}$ consolidándose el enlace $\mathrm{C}=\mathrm{S}$. Por otra parte, las señales correspondientes a los hidrógenos aromáticos no han sufrido un desplazamiento importante. La señal del hidrógeno amínico NH en el ligando (14,32 ppm) no es observada en el complejo de plomo (II), lo que corrobora que se produjo la desprotonación del grupo NH hidrazínico.

\section{Estudio computacional}

Se ha estudiado a nivel computacional por el método semiempírico PM3 debido a que el método presenta parametrización para el átomo de plomo ${ }^{5}$. Este método es muy popular para evaluar sistemas orgánicos (caso del ligando) y por ser muy preciso en cálculos de energía y longitud de enlace ${ }^{22}$.

En cada una de las estructuras de la figura 1: 1a), 1b) y 1c) se determinó la energía HOMOLUMO, la dureza $(\eta)$ energía a 0K y $298 \mathrm{~K}$ además del calor de formación. Para realizar un correcto modelado de las estructuras se tuvo que añadir el par libre presente en el átomo de plomo, ya que existe influencia de este par libre en la estereoquímica de los complejos de plomo (II) ${ }^{23,24}$ que presentan átomos dadores como él oxígeno ${ }^{25}$, azufre y selenio ${ }^{26}$. 


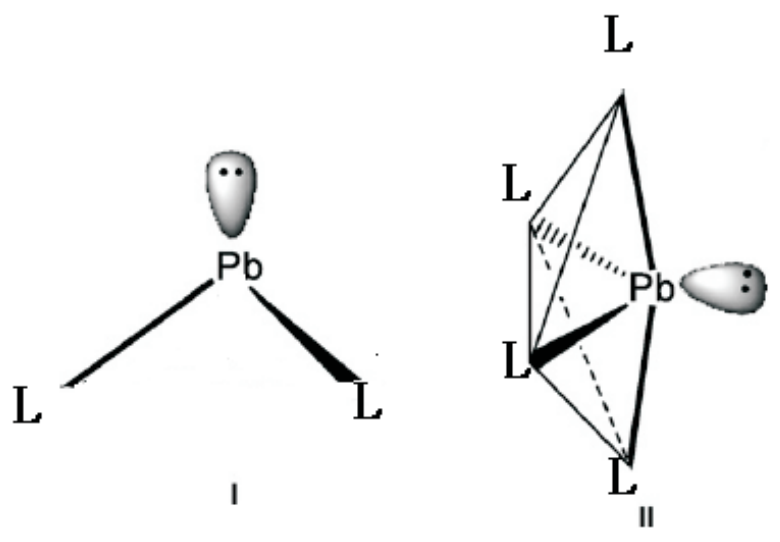

Figura 4. Geometrías de coordinación utilizadas para modelar el complejo de plomo (II).

En la figura 4 se muestra las geometrías de coordinación utilizadas para el modelar las tres posibilidades de coordinación vistas en la figura 1. El modelo I fue utilizado para modelar la estructura figura 1a y el modelo II para las estructuras 1b y 1c. Estas geometrías fueron introducidas al programa y optimizadas hasta obtener la mínima energía para cada uno de los casos.

En base a estas geometrías optimizadas de mínima energía se obtuvieron las siguientes propiedades mencionadas en la tabla 1.

Tabla 1. Energía total (a 0K, 298K), Calor de Formación, Dureza (n),Energía HOMO, Energía LUMO, Separación HOMO-LUMO para las tres posibilidades de coordinación del complejos de $\mathrm{Pb}$ (II).

\begin{tabular}{cccccccc}
\hline $\begin{array}{l}\text { ructuras } \\
\text { obables }\end{array}$ & $\begin{array}{c}\text { Energía total } \\
\mathbf{0 K}(\mathbf{K c a l} / \mathbf{m o l})\end{array}$ & $\begin{array}{c}\text { Energía total } \\
\mathbf{2 9 8 K}(\mathbf{K c a l} / \mathbf{m o l})\end{array}$ & $\begin{array}{c}\text { Calor formación } \\
(\mathbf{K c a l} / \mathbf{m o l})\end{array}$ & $\begin{array}{c}\text { HOMO } \\
(\mathbf{e V})\end{array}$ & $\begin{array}{c}\text { LUMO } \\
(\mathbf{e V})\end{array}$ & $\begin{array}{c}\text { Separación } \\
\text { HOMO-LUMO }\end{array}$ & $\begin{array}{c}\eta \\
\text { (dureza) }\end{array}$ \\
& & & & & & & \\
la & -116232.93 & -115755 & 266.962 & -8.332 & -2.583 & 5.75 & 2.87 \\
1b & -115836.92 & -115363 & 663.114 & -13.743 & -10.302 & 3.44 & 1.72 \\
1c & -116265.55 & -115810 & 234.347 & -8.392 & -2.024 & 6.37 & 3.18 \\
\hline
\end{tabular}

Se puede observar que de las tres estructuras propuestas, la estructura (1b) es la que menor estabilidad termodinámica y dureza química posee, por lo tanto se podría considerar una estructura bastante reactiva y poco estable, mientras que la estructura (1c) es la que posee mayor estabilidad termodinámica, buena separación HOMO-LUMO y mayor dureza de las tres opciones; cumpliendo con el Principio de Máxima Dureza de Pearson en el cual se asocia a la mayor separación HOMO-LUMO con la mayor estabilidad estructural y cinética ${ }^{14}$. 
Los datos experimentales muestran por medio de los espectros IR, $C^{13}\left(H^{1}\right)-A P T$ y $H^{1}-R M N$ que el ligando está totalmente desprotonado en el complejo, además de que sus puntos de coordinación son por medio del átomo de azufre y del nitrógeno hidrazínico (figura 1c).

En estudios estructurales con difracción de rayos $\mathrm{X}$ de complejos de plomo (II) tetracoordinados con tiosemicarbazona ${ }^{18}$ muestran la formación de enlace $\mathrm{Pb}-\mathrm{S}(2.730 \mathrm{~A})$ y $\mathrm{Pb}$ $\mathrm{N}(2.482 \AA)$, también los complejos de Pb (II) con 3-hexametileneiminiltiosemicarbazona ${ }^{19}$ exhiben puntos de coordinación en los átomos de azufre $\mathrm{Pb}-\mathrm{S}(2.7064 \AA$ ) y nitrógeno hidrazínico (2.628 A). Esto verificaría que la estructura (1c) es la que probablemente se formaría, ya que posee los mismos puntos de coordinación antes mencionados, además, los cálculos obtenidos muestran que dicha estructura posee mayor estabilidad y alta dureza global. El principio de ácido y base dura y blanda (HSAB) ${ }^{27}$ también nos puede ayudar a dilucidar cuál de las estructuras propuestas es la más probable. Tomando en cuenta que el catión de plomo $\mathrm{Pb}$ (II) es considerado como ácido intermedio y que el ligando posee dos zonas de coordinación en los átomos de azufre (blando) y del nitrógeno hidrazínico (duro) este catión puede interactuar con ambas zonas de coordinación o con las dos al mismo tiempo, dando un complejo bastante estable ${ }^{28}$.

Otro aspecto a tomar en cuenta es la influencia del par libre en los complejos de $\mathrm{Pb}$ (II), ya que el par libre provoca que aparezca una repulsión entre el par libre y los ligandos, mucho más fuerte, que entre los átomos del ligando.

Se propone entonces que debido a la influencia del par libre en la estereoquímica del complejo de plomo (II) la geometría tetraédrica no es la adecuada y que la geometría más probable será la geometría disfenoidal (balancín) o $\psi$ bipirámide trigonal ${ }^{26}$.

\section{CONCLUSIONES}

Las estructuras propuestas para el complejo de plomo (II) con el ligando 1,5 difenil-1,2,4 triazol-3-tiona han sido evaluadas a nivel semiempírico utilizando el método PM3 obteniendo que la estructura (3c) propuesta con el par libre adicionado al átomo de plomo, presentó mayor dureza y estabilidad termodinámica a comparación de las dos estructuras restantes (3a y 3b), cumpliendo así con el principio de máxima dureza de Pearson (PMH) y corroborando con lo encontrado experimentalmente.

La propuesta de una geometría tetraédrica para el complejo de plomo (II) parece no ser correcta, ya que la influencia del par libre en los complejos de plomo (II) con átomos dadores como el oxígeno, el azufre y el selenio, es importante y está avalada por la teoría de repulsión de pares de electrones de valencia (VSEPR) ya que el plomo no es un metal de transición, este estudio propone la geometría de balancín o $\psi$ bipirámide trigonal para el complejo Bis $(1,5-$ difenil-1,2, 4-triazol-3-tionato) plomo (II) $\left(\mathrm{Pb}(\mathrm{DTT})_{2}\right)$.

\section{REFERENCIAS}

1. Angulo-Cornejo J.; Serrano-Ruiz M.; Henning L. Síntesis y caracterización de 1,5 difenil-1, 2, 4-triazol-3-tiona (HDTT) y Bis (1,5-difenil-1, 2, 4-triazol-3-tionato) $\mathrm{Pb}$ (II) ((Pb(DTT) $\left.)_{2}\right)$ ). Rev. Soc. Quim 2003; 69(3): 131-142.

2. Clark, T.; Handbook of Computational Chemistry. New York: Wiley-Interscience; 1985.

3. Levine I.; Química Cuántica. 5ta edición. Madrid: Pearson Prentice Hall; 2001

4. Stewar J.J.; Optimization of parameters for semiempirical methods I, II Method and Applications. J. Compt. Chem 1989; 10 (2): 209-221.

5. Stewart J.J. Optimization of parameters for semiempirical methods. III. Extension of $\mathrm{PM} 3$ to $\mathrm{Be}, \mathrm{Mg}, \mathrm{Zn}, \mathrm{Ga}, \mathrm{Ge}, \mathrm{As}, \mathrm{Se}, \mathrm{Cd}, \mathrm{In}, \mathrm{Sn}, \mathrm{Sb}, \mathrm{Te}, \mathrm{Hg}, \mathrm{Tl}, \mathrm{Pb}$ and Bi. J. Compt. Chem 1991; 12 (3): 320-341. 
6. Cuevas G.; Cortes F. Introducción a la química computacional. México: Fondo de Cultura Económica; 2003.

7. Jensen F.; Introduction to Computational chemistry. $2^{\mathrm{a}}$ ed. England: Wiley; 2007.

8. Cramer Ch.J.; Essentials of Computational Chemistry. Theories and Models. $2^{\mathrm{a}}$ ed. England: Wiley; 2004.

9. Parr R.G. and Yang W.; Density-functional theory of atom and molecules. New York: Oxford Univ. Press; 1989.

10. Pauling L.; The nature of the chemical bond. Ithaca: Cornell University Press; 1960.

11. Pearson R.G.; Hard and Soft Acids and Bases. J Am Chem Soc 1963; 85 (22): 3533-3539.

12. Parr R.G and Pearson R.G.; Absolute hardness: Companion parameter to absolute electronegaty. J. Am. Chem. Soc., 1983; 105 (26): 7512-7516.

13. Koopmans Tyalling. Uber die Zuordnung von Wellenfunktionen und Eigenwerten Zudeneinzelnen Elektronen einer Atoms. Physica (Elsevier) 1934; 1-6:104-113.

14. Chattaraj P.K.; The Maximum Hardness Principle: an overview. Proc. Indian natn. Sci. Acad. 1996; (62) A, No6: 513-531.

15. Juan Andrés, Juan Beltrán, Química Teórica y Computacional. Madrid: Publicacions de la Universitat Jaume. D.L 2000.

16. Pearson R.G.; Maximum Chemical and Physical Hardness. J. Chem. Educ., 1999; 76 (2): 267.

17. Hypercube, Inc., Gainesville, Florida. USA. 1985-2007.

18. Castiñeiras A.; Domínguez R.; Bresolin L.; Bortoluzzi A.J.; Burrow R.A; Hörner M.; Synthesis and Crystal Structure of a Novel Neutral Polymeric Lead(II)thiosemicarbazonato Complex. Z. Naturforch. 1998; 53b: 81-86

19. Labisbal E.; Sousa A.; Castiñeiras A.; García-Vásquez J.A.; Romero J.; Douglas X. West. Spectral and structural studies of metal complexes of isatin 3hexamethyleneiminylthiosemicarbazone prepared electrochemi-cally. POLY. 2000; 19 : 1255-1262.

20. Dolphin D.; Wick A.; Tabulation of infrared Spectra Data. Wiley-Interscience Publication, John Wile and Sons, 1988.

21. Silverstein R. M.; Webster F.X.; Spectrometric Indentification of Organic Compounds, John Wiley and Sons, Inc. Sixth Editions, 1998.

22. Young D. C.; Computational Chemistry: A Practical Guide for Applying Techniques to Real World Problems. New York: John Wiley; 2001

23. Cotton A.F.; Wilkinson G.; Química inorgánica avanzada, $4^{\text {ta }}$ ed. México: Limusa; 2005.

24. Farzin. M. Synthesis and Characterization of lead (II) complexes with the 4Methoxybenzoyl Trifluoroacetonate Ligand. Z. Naturforch. 2009; 64b: 1027-1031.

25. Davidovich R. L.; Stavila V.; Stereochemistry of lead (II) complexes with oxygen donor ligands. Coord Chem Rev2009; 253: 1316-1352.

26. Davidovich R. L.; Stavila V.; Whitmire K.; Stereochemistry of lead (II) complexes containing sulfur and selenium donor atoms ligands. Coord Chem Rev 2010; 254: 2193 2226.

27. Pearson; R.G.; Hard R.G.; and Soft Acid and Bases HSAB Part I. Fundamentals Principles. J. Chem. Educ. 1968; 45(9): 581-586

28. Lawrance G.A.; Introduction to coordination Chemistry. $2^{\mathrm{a}}$ ed. New York: Wiley; 2010 